https://doi.org/10.46344/JBINO.2021.v10i02.36

\title{
CREATION OF AUTOMATED LOW-INTENSITY IRRIGATION SYSTEMS CONTROLLED THROUGH AN INTERNET CHANNEL IN AZERBAIJAN (ASMO)
}

Prof. Dr. Z.H. Aliev

Institute of Soil Science and Agrochemistry of the National Academy of Sciences of Azerbaijan

\section{ANNOTATION}

With the correct process management, low-intensity irrigation allows not only to drastically reduce water consumption for irrigation of agricultural crops, but also to provide the necessary microclimate for plants and the supply of water and fertilizers in the required amount directly to the root zone of plants, which contributes to the earlier entry of plants into the season fruiting and increased productivity while reducing water per unit of crop and reducing production costs. For this, we have developed a perfect safe technological system of automated irrigation based on IDAD with automated control of the controlled potential moisture supply of the plant, an optimized irrigation regime taking into account the controlled soil and agroclimatic parameters that affect the growth and development of the plant phase, while maintaining the ecological environment that contributes to obtaining a guaranteed and high-quality yield per unit area meeting the requirement of mi ovyh standards. The system of low-intensity irrigation created by the results of the study compared to traditional surface irrigation methods is more technologically demanding and in the remote areas from settlements it is difficult to provide qualified service; to ensure their possible efficiency, they require complex automation of the irrigation technological process.

Key words: low-intensity, automation, irrigation technique, regulatory, reference, data bank, controller, communications, object, sensors, etc. 


\section{INTRODUCTION}

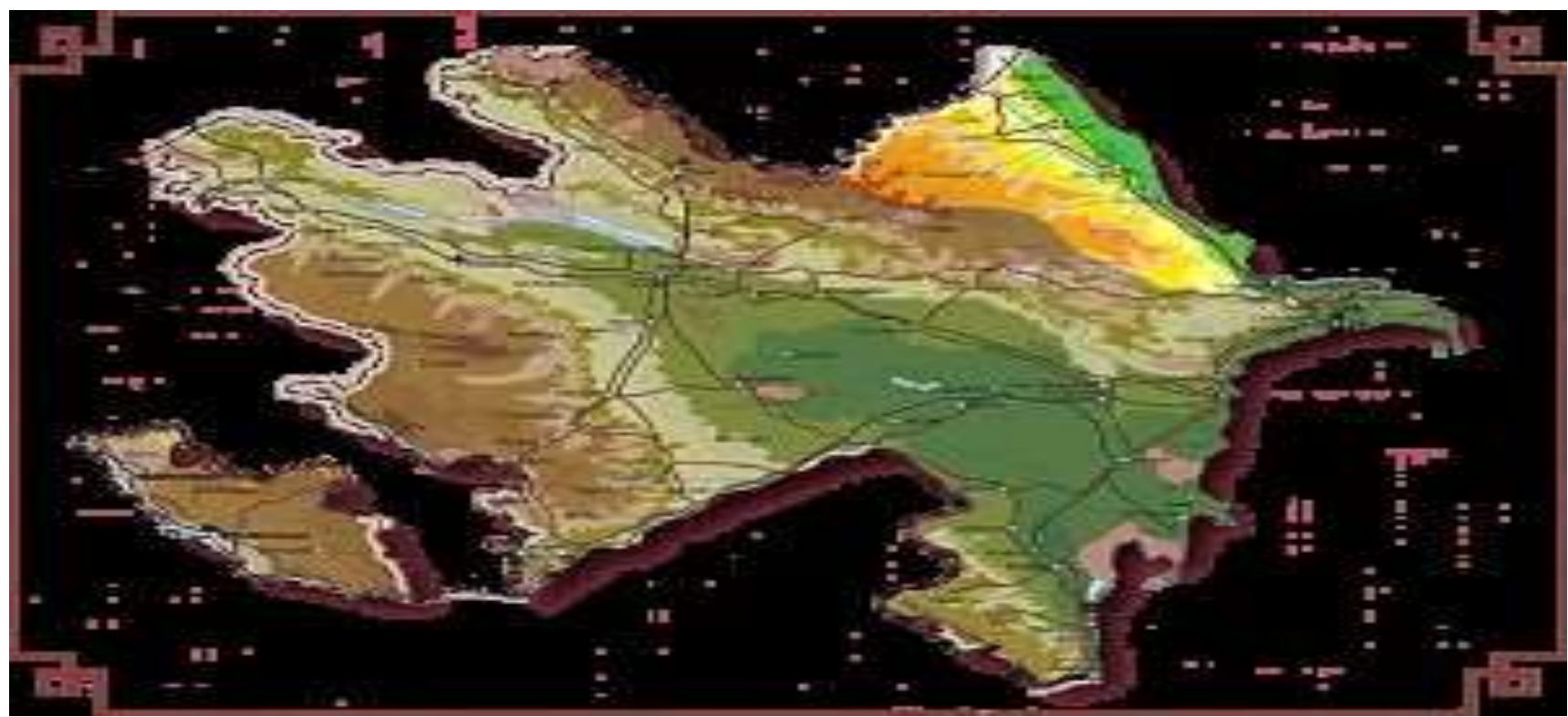

As you know, Azerbaijan is in difficult climatic and geographical conditions. Precipitation on the territory of the Republic is uneven, and in a number of mountain regions and insufficient for agricultural crops during their growing season, i.e. there is an acute shortage of water.

Most of the land suitable for agriculture is located in mountainous and foothill areas that require a special approach to irrigation techniques, i.e. when using conventional irrigation technologies, soil is washed off, surface runoff, erosion develops, etc.

One of the directions for solving this problem may be the transition to a lowintensity water supply in accordance with the needs of agricultural crops during their growing season. In this connection, the task of the study was set to develop and create a system of low-intensity irrigation, which is automatically controlled in the conditions of mountain farming, providing the requirements of the study.

The purpose of the study: is the development and implementation of an automated system of low-intensity irrigation (ASMO) for work in the mountains and foothill region of Azerbaijan.

Tasks of the research: creation on the basis of remote measurement parameters and computer technology with the organization of data exchange channels via the Internet, which determine the technological process of the plants, which form the structure and intensity of moisture supply to the soil and control the quality of the soil in irrigation fields, as well as the development of agricultural crops

Research methodology: to solve this problem, the questions of creating a typical automated system of low-intensity irrigation for mountain and foothill regions of the republic are considered.

Study paths and discussion of results

Considering the heterogeneity of land reclamation conditions for cultivating agricultural crops on farms and the great dispersion of climatic and geographical conditions, which is typical for mountainous and foothill areas, when creating an ASMO, a decentralized automated system linked to individual farms and centralized operational control 
of local systems for Internet channels. [2.6].

Monitoring and control of the operational and technological regime of the agrotechnical process is carried out on the basis of instrumental measurement data on parameters characterizing the composition and condition of the soil, atmosphere, phase of plant development and ongoing agrotechnical measures recorded in real time in the Databank. [7]. The data bank (DB) is formed from the following sections:

a) Normative and reference - including a set of parameters with which to bind to any variations of field characteristics encountered in practice: to the types of their soil and ecological conditions, types of cultivated crops, types and modifications of equipment, etc. These data are constantly stored in the computer.

b) Seasonal conditionally constant including sets of parameters with the help of which specific characteristics of each field are taken into account, which usually do not change during the growing season: area, topography, type, waterphysical properties of the soil, the depth and mineralization of groundwater, the type and variety of crops grown, prohibited equipment, etc. This information is entered into the database with the possibility of its correction.

c) Operational - necessary for the operational planning of technological operations, taking into account current situations in the fields.

Its composition is divided into independent of the farmer and feedback data on the implementation of planned operations. The first include: temperature, humidity and precipitation, the actual time of the onset of the phases of plant development, the appearance of pests in the fields, etc. Feedback data is the executive (actual) dates, norms for irrigation and the results of control measurements of soil moisture, the timing, form and dose of fertilizer application and the data of control measurements.

The formation of the database and work with data is carried out by programs developed in the programming languages Visual FoxPro and Clipper.

Data is entered into the Databank by recordings in files of the developed structure (See the section "Information Support") either automatically when measuring in real time, or according to a schedule or request.

The purpose of automation of irrigation management is to ensure, in specific natural and agrophysical conditions, the maximum yield of agricultural crops at the smallest irrigation water consumption and minimization of negative impacts on land fertility and the environment, which is ensured by solving the following tasks:

1. Information and reference

2. Operational information

3. Operations Manager

\section{Applied agricultural tasks}

5.Preparation of information reports on the progress and results of irrigation.

Information and reference, operational information, applied agrotechnical tasks and the preparation of information reports are carried out by software tools developed in the Clipper programming languages. and Visual FoxPro; tasks of the operational management complex - in the $\mathrm{C}++$ programming language.

1. The complex of tasks of operational planning and management for ASMO facilities

\subsection{Data collection from objects}

1.1.1. For operational monitoring of weather conditions necessary to solve the 
problems of planning and operational management of irrigation of fields of agricultural crops, measurement sensors with transducers for telemetry from reading measurements are installed on the hydrometeorological station: (see. one).

a) wind speed - $V$ analog signal (TIT) with a period of recording parameter values in a cycle of $30 \mathrm{~min}$.

b) air temperature - tv, analog signal (TIT) with a period of recording parameter values in a cycle of 30 minutes.

c) air humidity - $W_{B}$, an analog signal (TIT) with a period of recording parameter values in a cycle of $30 \mathrm{~min}$.
The reading of the parameter values in the telemetric code is carried out by an intelligent object controller ( $\mathrm{KO}$ ) installed in the transformer station through a radio channel that communicates with the transducers. The signals measured by the telemetry codes (see the "Information Support" section) are processed in primary digital code, averaged, and written to RAM, where they are stored until they are counted by the communication controller (CS) installed in the operational control room of the technological process (ASMO) - operator. $[2,3]$.

The structural-functional diagram of the industrial control system and the automatic control system of irrigation developed by us is shown in Fig. 1 and 2 .

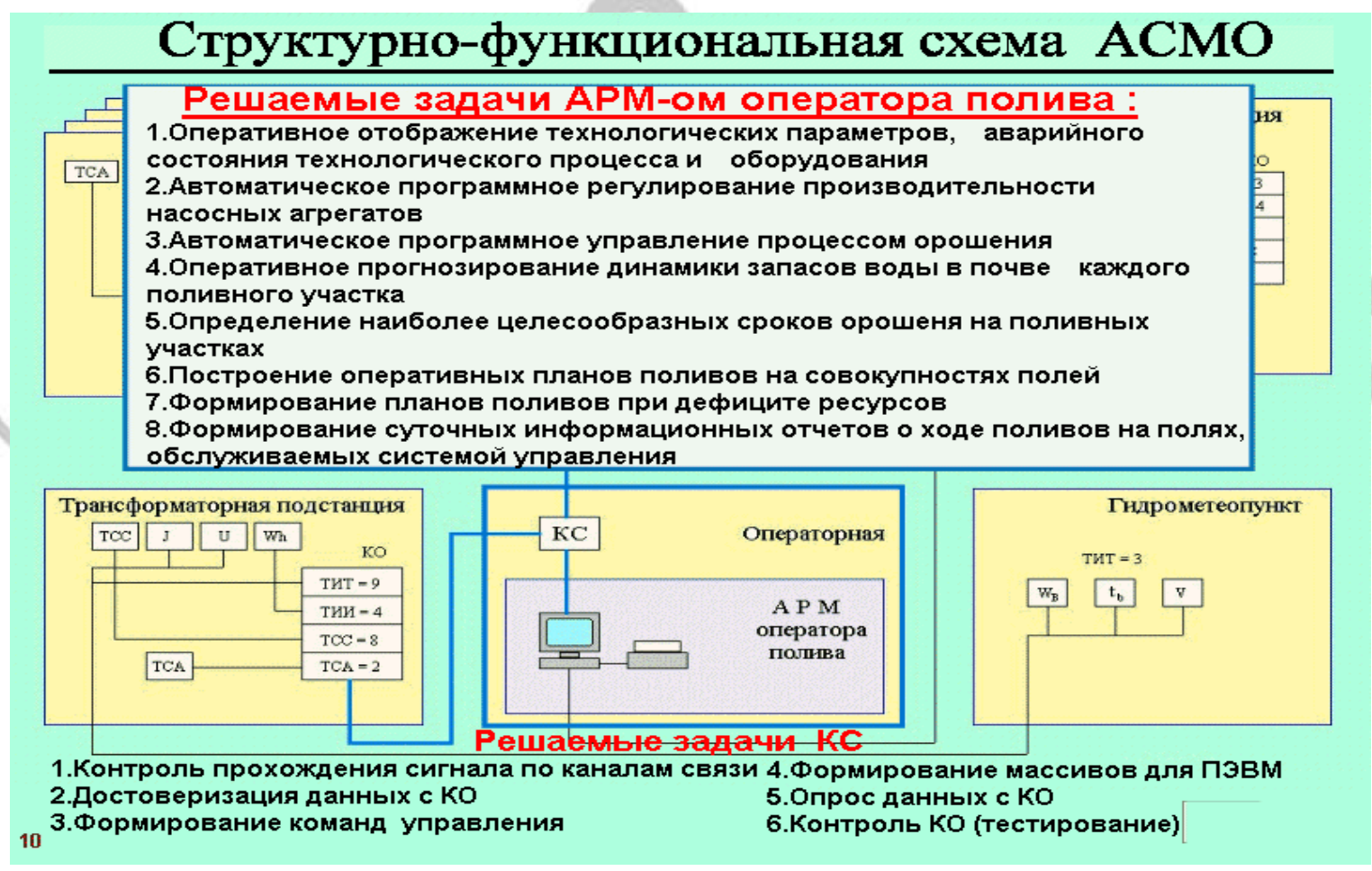

Fig. 1. Structural and functional diagram of the industrial control system for irrigation. 


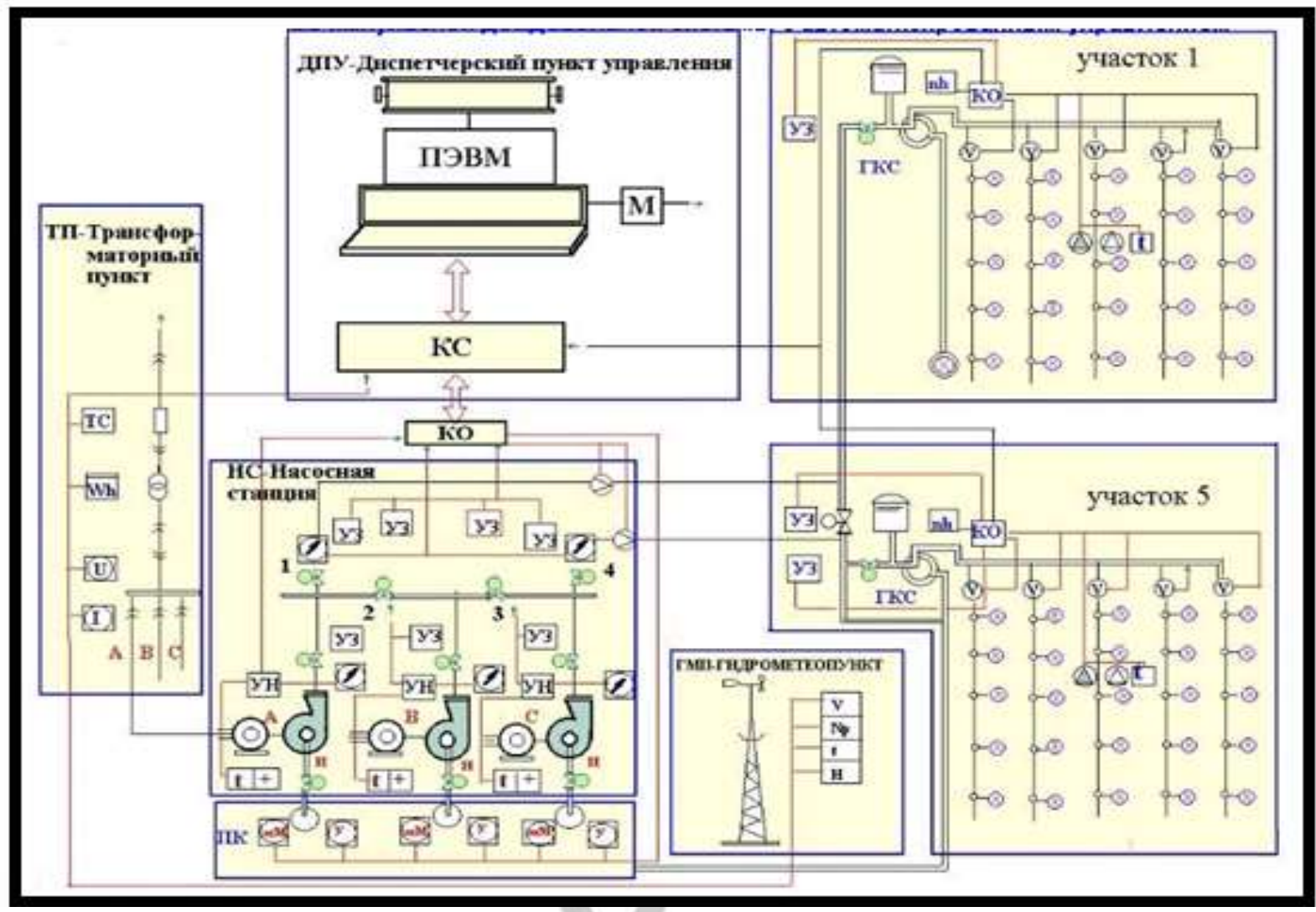

Fig. 2. Schematic diagram of a pulsed sprinkler automated control systems

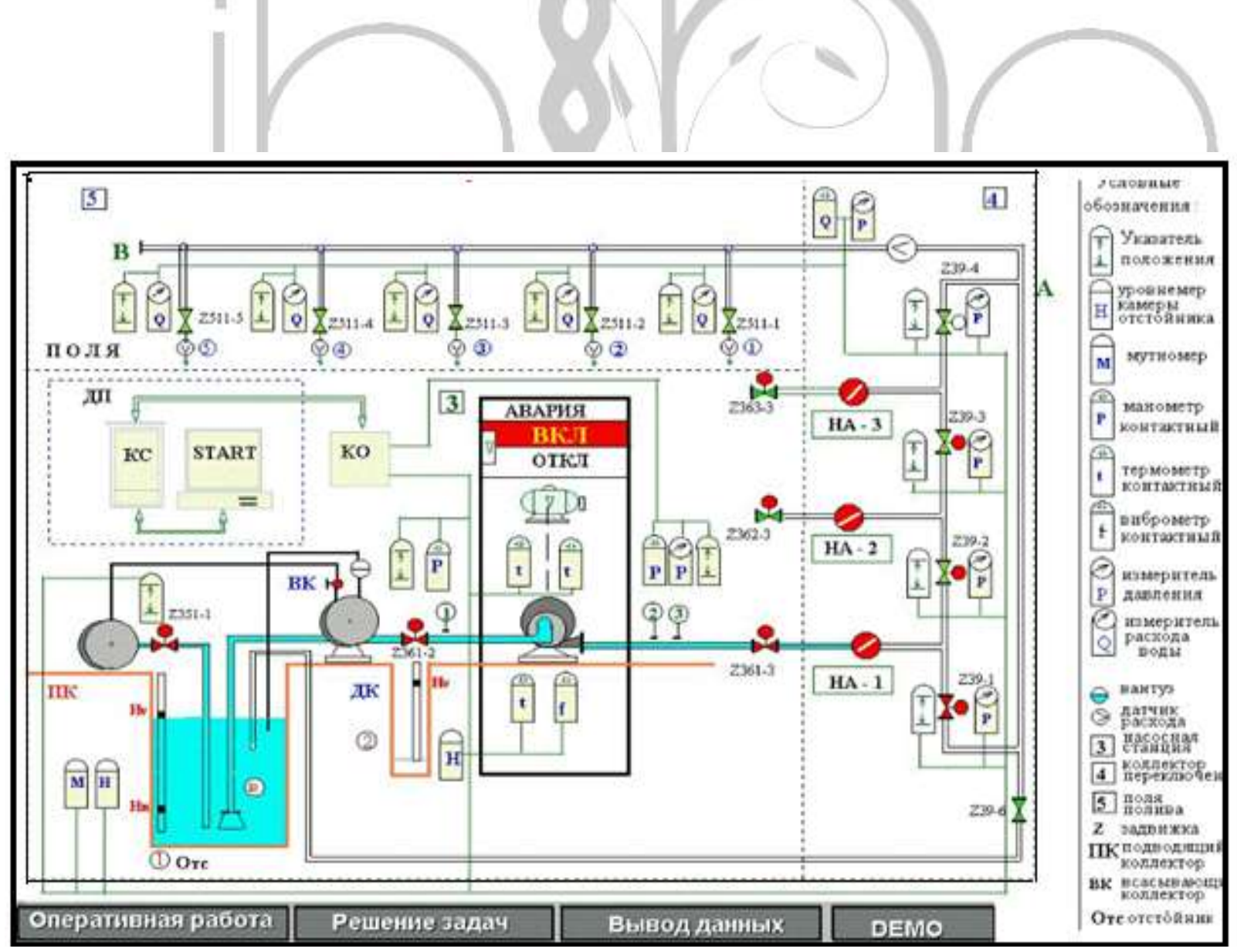

Fig. 3 Starting the pump motor 


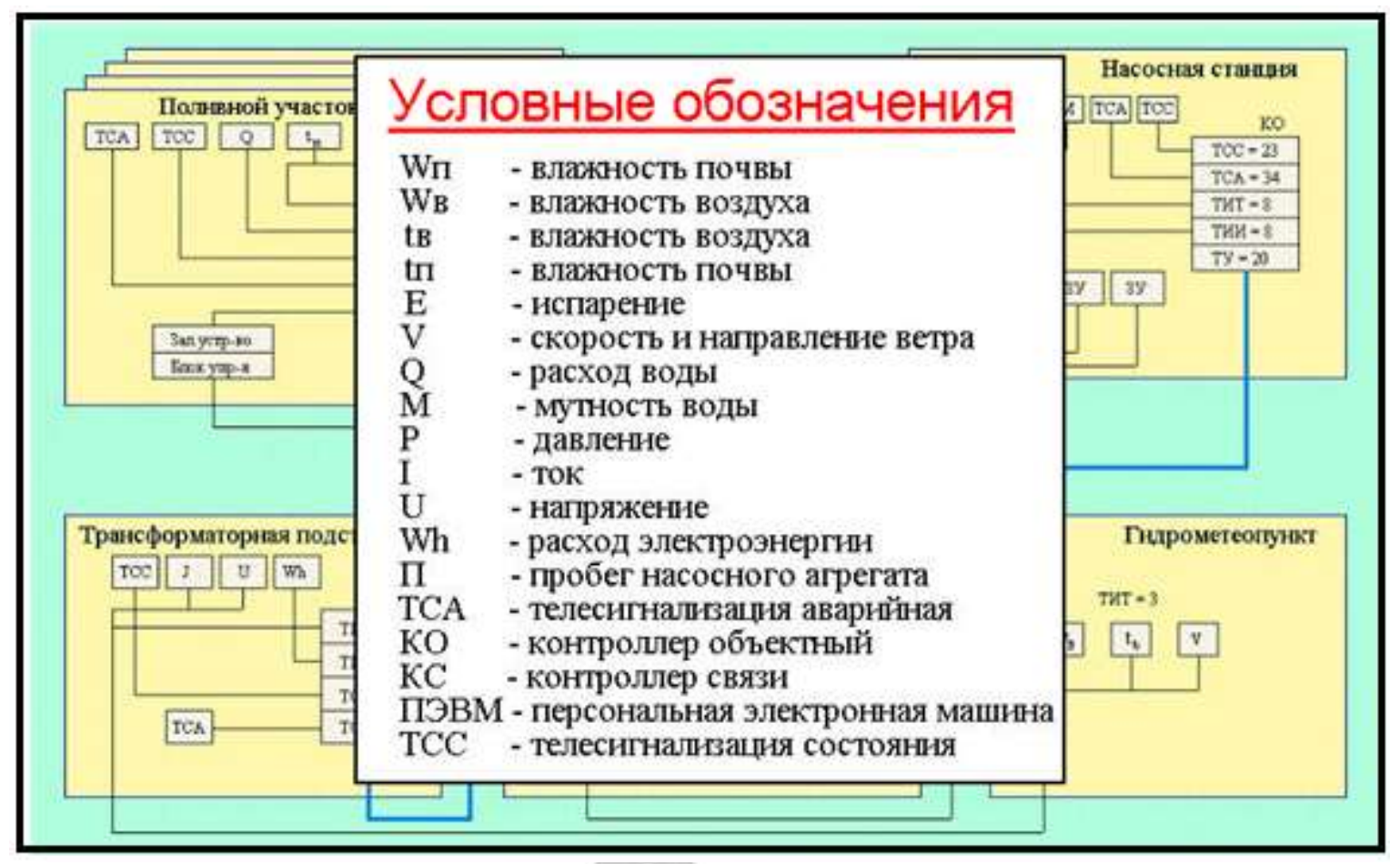

Fig. 4. Structural and functional diagram of industrial control system for irrigation

1.2. For the control and management of power supply facilities ASMO and metering of power consumption at the transformer point (TP) (see the structural and schematic diagram of the automatic process control system of irrigation) transducers are installed:

a) measuring the voltage at the input to the TP - U (analog signal (TIT);

b) consumer load measurements - I U (analog signal (TIT);

c) accounting for energy consumption Wh (integrated discrete signal - TIl;

d) control the position of the switches

d) (inclusion - disconnection of electric consumers) - SS

e) (discrete signal position TCC).

The report of parameter values in the telemetric code is carried out by an intelligent object controller ( $\mathrm{KO}$ ) via local wired communication channels and, after their initial processing and averaging, are recorded in the $\mathrm{KO}$ random access memory.

1.3. To monitor and control the technological process of water intake, sedimentation tanks (treatment facilities) and a pumping station (devices for increasing water pressure in pipelines), transducers are installed (see structural and functional diagram):

a) turbidity of water in sedimentation tanks - $M$; (analog TIT signal read in a cycle of $30 \mathrm{~min})$;

b) the water level in the settling chambers - N; (analog TIT signal read in a cycle of 30 minutes);

c) the pressure of water - $P$ installed on the discharge pumps, prefabricated and distribution collectors; (analog TIT signal read in a cycle of $30 \mathrm{~min}$ );

d) measuring the load of electric motors - I; (analog TIT signal read in a cycle of 30 minutes); 
d) the position of the valves - $\mathrm{PZ}$; (discrete signal TSS, read in a cycle of $1 \mathrm{~s}$ );

f) the provisions of the power switches VP; (discrete signal TCC, read in a cycle of $1 \mathrm{~s})$;

g) alarm - AC; (discrete TCA signal read in a cycle of $1 \mathrm{~s}$, priority);

h) measuring the flow rate supplied by pumps and in the distribution pipe - Q; (integrated TIl signal processed in a cycle of 1 hour). (see fig. 1)

1.4 .. Monitoring of the soil condition and process control of irrigation is carried out on individual fields of irrigation based on measurements of agrophysical and technological parameters by transducers:

a) soil moisture of the VLP; - (analog TIT signal recorded in a cycle of $30 \mathrm{~min}$ );

b) evaporation of water from the soil surface - ISP - (analog signal TIT recorded in a cycle of $30 \mathrm{~min})$;

c) soil temperature - to; - (analog TIT signal recorded in a cycle of $30 \mathrm{~min}$ );

d) the flow rate of water for irrigation through the distribution pipeline of the site - Q - (integrated signal recorded in a cycle of 30 minutes);

d) switching on the GCS - a discrete signal read in a cycle of $30 \mathrm{~s}$;

f) the position of the switching valves; (discrete position signal TCC - read cycle $30 \mathrm{~s})$.

The signal report in the telemetric code is carried out by an intelligent object controller of the field via radio channels of communication and, after their initial processing and averaging by the processor, are written into the RAM

2. Entering operational data into a computer and forming a database (HBS)
The data recorded in the operative memory of object controllers (KOs) is counted programmatically via radio and wire communication channels by a communication controller connected to a control room computer (DP) (see Schematic diagram of a lowintensity irrigation system with automated control Fig. 4.), according to the specified regulations and are recorded in its RAM in the structure of the telemetry file (see. Information support).

The computer, using exchange programs, reads data from the RAM of the CS, transcodes it and writes it to the operational database, from which it displays them in real time for display on mnemonic diagrams, and after linearization and averaging, the data by their codes are programmatically recorded in the storage databases, the structures of which are given in information support, and this forms the Data Bank of the ASMO complex of tasks. $[3,7]$.

\section{findings}

The newly created system of pulse selfoscillating sprinkling irrigation operating with an automated control unit has a fundamental difference from its predecessors in that it allows you to control the irrigation process in the fields remotely from the operator, where the automated system is tied according to the operational-technological mode to individual farms and centralized operational control the work of local systems on the Internet.

\section{Literary sources:}

1.Aliev B.H., Aliev Z.H and others Techniques and technology few intensive irrigations in condition of the mountain 
region Azerbaijan. Publishers "Elm", Baku, 1999, p. 220.

2.Aliev B.H., Aliev Z.H. The premises about the most important problem of the agriculture in provision water resource mountain and foothill regions Azerbaijan, J. AAS, \# 1-3, Baku, 2007, p. 179-182.

3.Aliev B.H., Aliev Z.H. The premises of the decision of the problems moisture provides agriculture cultures production in mountain and foothill region Azerbaijan. The works SRI "Erosions and Irrigations". Baku, 1999, p. 125-129.

4. Aliev B.H., Aliev Z.H. Zoning of the territory of the Republic of Azerbaijan for the selection of advanced irrigation technologies. Monograph, Zia-Nurlan Publishing House, Baku, 2001, p. 249.

5.Huseynov G.M. Ways to increase the efficiency of irrigated land use, improve technology and methods of irrigation of agricultural crops in Azerbaijan. Competition degree report. C. S. D. based on work, Baku, 1969, p.30.
6. International Center for Agricultural Research in arid regions in arid regions (ICARDA) Irrigation regime and monitoring technique. Edited by $U$. Umarov and A. Karimov. Taraz: IC "AQUA", 2002, $128 \mathrm{~s}$.

7.V.F. Nosenko Irrigation in the mountains. Publishing House Kolos Moscow 1981.143 p.

\section{Summary}

To articles Prof., Dr. Aliyeva Z.H. on the topic: Creation of automated lowintensity irrigation systems (ASMO) managed through the Internet channel in the conditions of Azerbaijan

The system of low-intensity irrigation created by the results of the study compared to traditional surface irrigation methods is more technologically demanding and in the remote areas from settlements it is difficult to provide qualified service; to ensure their possible efficiency, they require complex automation of the irrigation technological process. 